PHYSICAL REVIEW D 94, 059903(E) (2016)

\title{
Erratum: Preserving the validity of the two-Higgs-doublet model up to the Planck scale \\ [Phys. Rev. D 92, 033003 (2015)]
}

Pedro Ferreira, Howard Haber, and Edward Santos

(Received 12 September 2016; published 26 September 2016)

DOI: 10.1103/PhysRevD.94.059903

There was a typographical error in Eq. (A28). The corrected equation is as follows:

$$
\begin{aligned}
\mathcal{D} Z_{a \bar{b} c \bar{d}}= & 4 Z_{a \bar{b} e \bar{f}} Z_{c \bar{d} f \bar{e}}+2 Z_{a \bar{f} c \bar{e}} Z_{f \bar{b} e \bar{d}}+2 Z_{a \bar{f} e \bar{d}} Z_{f \bar{b} c \bar{e}}+2 Z_{a \bar{b} e \bar{f}} Z_{c \bar{e} f \bar{d}}+2 Z_{a \bar{e} f \bar{b}} Z_{c \bar{d} e \bar{f}} \\
& -\left(3 g^{\prime 2}+9 g^{2}\right) Z_{a \bar{b} c \bar{d}}+\frac{3}{4}\left(3 g^{4}-2 g^{\prime 2} g^{2}+g^{\prime 4}\right) \delta_{a \bar{b}} \delta_{c \bar{d}}+\left(3 g^{\prime 2} g^{2}\right) \delta_{a \bar{d}} \delta_{c \bar{b}}-4 N_{c} \operatorname{Tr}\left[\eta_{a}^{Q} \eta_{\bar{b}}^{Q^{\dagger}} \eta_{c}^{Q} \eta_{\bar{d}}^{Q^{\dagger}}\right] \\
& +4\left(\operatorname{Tr}\left[\eta_{\bar{e}}^{Q^{\dagger} \dagger} \eta_{a}^{Q}\right] Z_{e \bar{b} c \bar{d}}+\operatorname{Tr}\left[\eta_{\bar{b}}^{Q^{\dagger}} \eta_{\bar{e}}^{Q}\right] Z_{a \bar{e} c \bar{d}}+\operatorname{Tr}\left[\eta_{\overline{\bar{e}}}^{Q^{\dagger}} \eta_{\bar{c}}^{Q}\right] Z_{a \bar{b} e \bar{d}}+\operatorname{Tr}\left[\eta_{\bar{d}}^{Q_{\bar{d}}^{\dagger}} \eta_{\bar{e}}^{Q}\right] Z_{a \bar{b} \bar{c} \bar{e}}\right) .
\end{aligned}
$$

